\title{
The contribution of morphine sulfate to the development of necrotizing enterocolitis in preterm infants: a matched case- control study
}

\author{
Zlatan Zvizdic ${ }^{1}$, Emir Milisic ${ }^{1}$, Asmir Jonuzi ${ }^{1}$, Sabina Terzic ${ }^{2}$, Denisa Zvizdic ${ }^{3}$ \\ ${ }^{1}$ Clinic of Pediatric Surgery, ${ }^{2}$ Pediatric Clinic, Neonatal Intensive Care Unit, ${ }^{3}$ Eye Clinic, Department of Pediatric \\ Ophtalmology, University Clinical Center Sarajevo, Sarajevo, Bosnia and Herzegovina. E-mail: zlatan.zvizdic@gmail.com \\ Received: 2nd August 2018, Revised: 2nd October 2018, 21st November 2018, Accepted: 15th December \\ 2018
}

SUMMARY: Zvizdic Z, Milisic E, Jonuzi A, Terzic S, Zvizdic D. The contribution of morphine sulfate to the development of necrotizing enterocolitis in preterm infants: a matched case-control study. Turk J Pediatr 2019; 61: 513-519.

\begin{abstract}
The aim of the study was to determine whether morphine sulfate administration is associated with an increased risk of necrotizing enterocolitis (NEC) in preterm infants supported by mechanical ventilation due to respiratory failure. The matched case-control retrospective study was carried out at the Neonatal Intensive Care Unit (NICU) of the University Clinical Center Sarajevo, on 122 preterm infants classified into total NEC group and control group. The total NEC group was further divided into medical NEC and surgical NEC subgroups. The association between the use and duration of morphine sulfate infusion and the development of NEC was evaluated in both unadjusted and adjusted analysis. Preterm infants who developed NEC were on mechanical ventilation more frequently compared to premature infants without signs of NEC (MannWhitney $U$ test; $p=0.0031$ ). A positive correlation between the frequency of receiving morphine sulfate and the development of NEC was observed (Chi square test of independence; $p=0.0001$ ). The risk of NEC in preterm infants was increased by the use of morphine sulfate. Validation of this observation in other populations is warranted.
\end{abstract}

Key words: morphine sulfate, opioids, mechanical ventilation, necrotizing enterocolitis, preterm infants.

It is well recognized that the etiology of necrotizing enterocolitis (NEC) is multifactorial ${ }^{1-3}$ but exact pathophysiological mechanisms remain unclear. ${ }^{4}$ Many low birth weight preterm newborns and sick full-term newborns, including those with NEC, require respiratory support using endotracheal intubation and mechanical ventilation. ${ }^{5}$ The reduction of pain in newborns on mechanical ventilation is a crucial part of the supportive treatment due to the wellknown fact that this mode of treatment is accompanied by a physiological, biochemical and clinical responses to pain and stress. Pain and stress may have a negative interaction with mechanical ventilation, leading to unsynchronized breathing and suboptimal ventilation with possible clinical instability, changes in heart and respiratory rates and changes in intracranial pressure, blood pressure and oxygen saturation. ${ }^{6,7}$ However, there are still no harmonized clinical guides for the use of analgesics and sedatives in critically ill infants, ${ }^{8}$ and most recommendations are based on experiences with adult patients. ${ }^{7-9}$ Morphine sulfate is one of the opioid analgesic used for sedation and analgesia in newborns, ${ }^{12}$ but there is insufficient evidence to recommend routine use of opioids in newborns on mechanical ventilation. ${ }^{7}$ In this study, we hypothesized that the frequency of NEC in preterm infants who require mechanical ventilation would be increased with an increase in the number of days of receiving morphine sulfate. 


\section{Material and Methods}

This matched case-control retrospective study was carried out at the Neonatal Intensive Care Unit (NICU) of University Clinical Center Sarajevo (UCCS), on 122 preterm infants classified into total NEC group and control group. NEC group included 51 consecutive preterm infants admitted to the NICU of UCCS and treated for NEC during a period of 5 years, from January 2008 to December 2012. The control group included 71 apparently healthy preterm infants. Gestational age was determined by early ultrasound and recorded as completed weeks. This study examined whether morphine sulfate administration was associated with an increased risk of NEC in preterm infants supported by mechanical ventilation due to respiratory failure. For the purposes of this analysis, a diagnosis of NEC was made based on the presence of clinical, radiological and/or histopathological evidence that fulfilled the criteria of Bell's ${ }^{13}$ as well as Walsh's modification of these criteria. ${ }^{14}$ Medical NEC was defined as the presence of radiological signs of pneumatosis intestinalis and when the disease is treated with antibiotics for more than two days. Surgical NEC was defined as any surgical treatment. Fifty-one preterm infants met the criteria for NEC in this analysis. The infants' medical records were reviewed daily for medical course information until hospital discharge or death of infant. A standardized format was used for data collection. In accordance with the Helsinki declaration, the Institutional Review Board (IRB), and the Independent Ethics Committee of UCCS approved all aspects of this study. All medical record data included in this study were used after obtaining written informed consent from the patient's parents.

The study was approved by the Institutional Review Board of University Clinical Center Sarajevo (Number: 0305-11118 from 14th April 2010).

\section{Statistical analysis}

To compare the frequency of mechanical ventilation and administration of morphine sulfate in premature infants who did and did not develop NEC, we used the MannWhitney $\mathrm{U}$ test for continuous variables and chi-square test of independence for categorical variables. Statistical level of $95 \% \quad(p<0.05)$ was considered significant for all performed tests. Statistical analysis was performed using SPSS 16.0 (SPSS Inc, Chicago, IL, USA).

\section{Results}

Among infants with NEC, medical NEC was diagnosed in 30 patients $(58.8 \%)$, while 21 patients were classified in the group of surgical NEC (41.2\%).

Table I provides demographic and clinical information for the 122 preterm infants in the study.

Postnatal age at time of appearance of NEC was 18.2 days (SD 12.8) (2-57 days); $49 \%$ of infants were older than 2 weeks. All subjects were breast fed in majority of cases (40/51). We always used freshly expressed breast milk. No baby was fed by donor breast milk. Therefore, we did not notice any differences between study groups related to the type of enteral feed. In one patient, NEC developed before starting enteral feeding.

The most common gastrointestinal symptoms in total NEC group were: Abdominal distension in $89 \%$, macroscopic or microscopic blood in the stool in $56.9 \%$ and increasing gastric residuum in $46 \%$ of cases.

During mechanical ventilation of our patients we used morphine sulfate as an analgesic agent at the infusion dosage of $20 \mu \mathrm{g} / \mathrm{kg} / \mathrm{h}$. In selected cases, where morphine was insufficient, we used midazolam as a sedation at the dosage of $1-2 \mu \mathrm{g} / \mathrm{kg} / / \mathrm{min}$. This was a case in $5(9.8 \%)$ of the ventilated infants.

The average mechanical ventilator days in all preterm infants with NEC (both medical and surgical NEC) $(n=51)$, in the medical NEC group $(n=30)$, in the surgical NEC group $(n=21)$, and in the healthy control group $(n=71)$ are presented. Our results indicate that the mean number of days of mechanical ventilation in the NEC group of preterm infants was 8.0 days ( 0.5 to 44$)$, in the medical NEC group 8.0 days (5-31), and in the surgical NEC group 11.0 days (1-44). The mean number of days of mechanical ventilation in the control 
荘

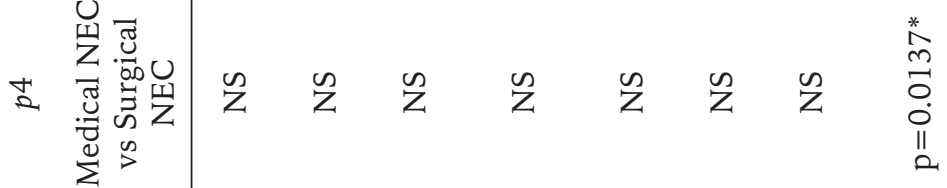

㟧

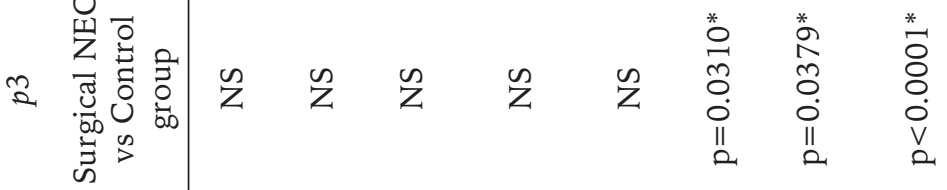

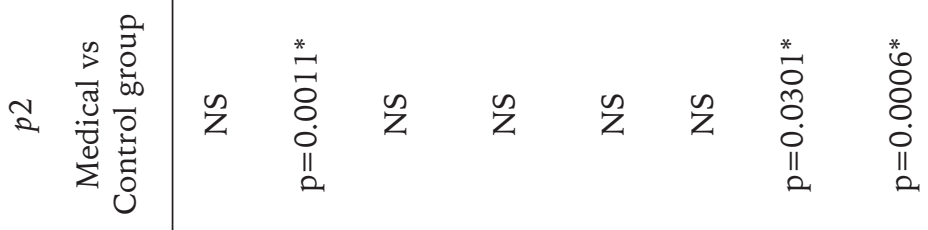

$>$ 刍

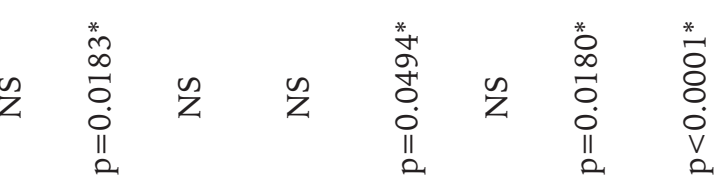

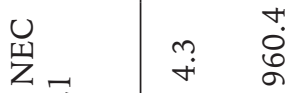

픈

50

is

U

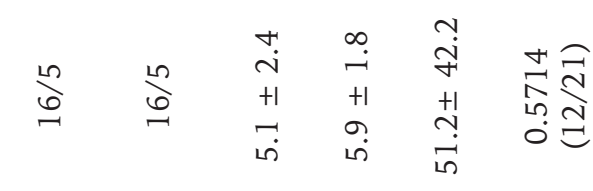

전

:ृ

$<$

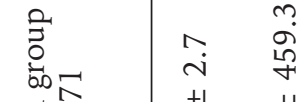

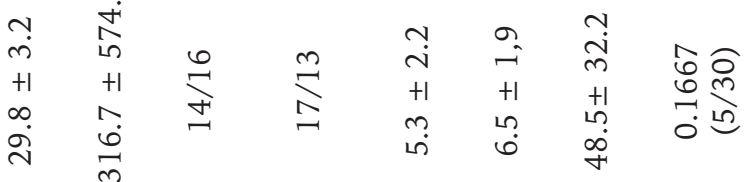

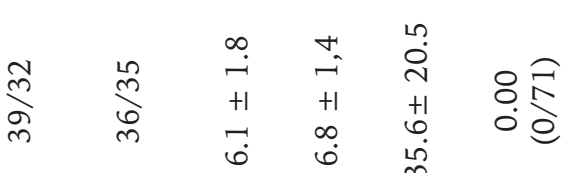

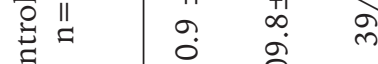

กे

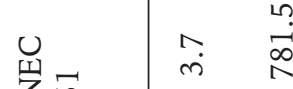

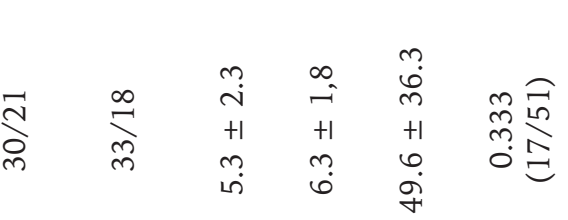

퓽

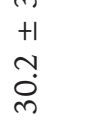

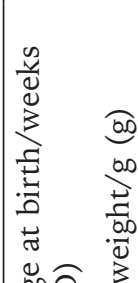

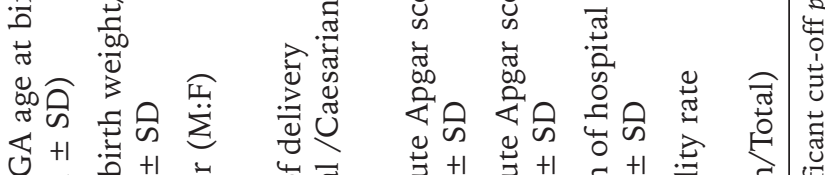

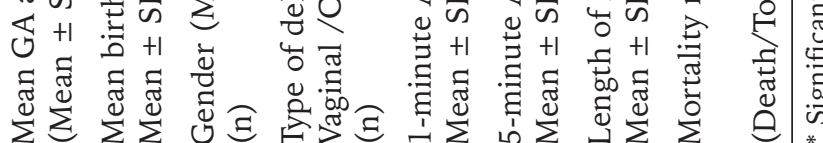


group of preterm infants was 3.0 days (1-14).

A significant difference in the number of days of mechanical ventilation in infants in the NEC group $(M d=8, n=43)$ compared to the control group was found (MannWhitney $U$ test, $(M d=3, n=22), U=262.00$, $z=2.955, p=0.0031$. In addition, a statistically significant difference in the mean number of days of mechanical ventilation in the medical NEC group $(M d=8, n=25)$ compared with the control group was observed $(M d=3, n=22)$, $\mathrm{U}=150.50, \mathrm{z}=2.681, \mathrm{p}=0.0073$. There was also a statistically significant difference between the surgical NEC group $(\mathrm{Md}=11, \mathrm{n}=19)$ and control group $(M d=3, n=22), U=111.50, z$ $=2.604, p=0.0092$. In contrast, no significant difference was found in the number of days of mechanical ventilation between the medical and surgical NEC groups $(p=0.4126)$.

Our data indicate that $72.5 \%(37 / 51)$ of premature infants with NEC received morphine sulfate. Further analysis showed that $63.3 \%$ $(19 / 30)$ premature infants with medical NEC and $85.7 \%(18 / 21)$ infants with surgical NEC received morphine sulfate. In the control group of premature infants, morphine sulfate was received by $19.7 \%$ patients $(14 / 71)$.

Chi square test of independence (with Yates' correction for continuity) showed a significant correlation between the frequency of received of morphine sulfate and the development of NEC in patients with NEC compared to the control group $\chi^{2}(1, \mathrm{n}=122)=31.914$, $\mathrm{p}=0.0001$. A statistically significant difference was observed between the medical NEC group compared to the control group $\chi^{2}(1, \mathrm{n}=101)$ $=16.308, \mathrm{p}=0.0001$, and between the surgical NEC group compared with the control group $\chi 2(1, \mathrm{n}=92)=30.795, \mathrm{p}=0.0001$. In contrast, no significant difference was found between two NEC groups regarding the frequency of receiving morphine sulfate $(\mathrm{p}=0.1106)$.

The analysis of the frequency of received inotropes revealed a statistically significant association between administrations of inotropes in infants with NEC compared with control group. In the total NEC group, $64.7 \%$ $(33 / 51)$ NEC patients received inotropes compared with only $12.7 \%(9 / 71)$ in the control group $(p=0.001)$. Preterm infants with medical NEC received inotropes in $56.7 \%$ $(17 / 30)$ while infants in surgical group received inotropes in $76.2 \%(16 / 21)(p=0.2550)$.

\section{Discussion}

It is well known that extremely low (ELBW) and very low birth weight infants (VLBW) have an increased incidence of respiratory distress syndrome (RDS) requiring mechanical ventilation. $^{15}$ Also, for many premature infants, especially those with ELBW and VLBW, the deterioration of the respiratory system, in the form of an increase in required respiratory support and decreased $\mathrm{PO} 2$ and O2 saturation, may be the early clinical sign of the development of NEC, ${ }^{15}$ although the cause of this finding has not been sufficiently explained. Dolgin et al. ${ }^{15}$ found that exposure to extended periods of mechanical ventilation can lead to an increased number of nosocomial infections thereby increasing the risk of NEC. The results of our study have shown that premature infants with NEC required a longer period of mechanical ventilation compared to the control group. This statistically larger number of days of mechanical ventilation was observed both in the medical NEC group and the surgical NEC group.

Since previous studies, performed at the end of 1990s, demonstrated that intravenous administration of morphine sulfate improves synchronization of spontaneous and mechanical ventilation derived breathing in premature infants, as well as that continuous infusions of morphine sulfate reduces the incidence of poor neurodevelopmental outcome in premature infants who require respiratory support through mechanical ventilation, administering morphine sulfate has become a common practice in neonatal intensive care units. ${ }^{16,17}$ With time, it was recognized that the use of morphine sulfate has no effect on reducing the frequency of intraventricular hemorrhage nor its clinical outcome. ${ }^{18}$ Furthermore, the observation of the side and adverse effects of morphine sulfate in the form of hypertension, bradycardia, convulsions, intestinal obstruction, delaying the start time of enteral feeding and particularly the reduction of gastrointestinal motility, ${ }^{19}$ has lead to questioning the routine administration 
of morphine sulfate in premature infants undergoing mechanical respiratory support. ${ }^{20}$ Hallstrom et al. ${ }^{21}$ studied the risk factors of NEC and noted that the length of the duration of administration of morphine sulfate has a significant impact on the development of NEC. To the best of our knowledge, the study mentioned above was the only study to evaluate the influence of morphine sulfate on the development of NEC. The effect of morphine sulfate on NEC development can be explained by the knowledge that morphine sulfate reduces gastrointestinal motility, which is already disturbed in premature infants, allowing a prolonged contact of the bacterial strains from food substrates with the intestine wall, thereby allowing bacterial translocation and development of this devastating disease. The results of our research on the effect of administration of morphine sulfate in developing NEC have shown that preterm infants with NEC (both medical and surgical NEC groups) had a significantly higher number of days receiving morphine sulfate before demonstrating symptoms of the disease, compared to the control group. We also recorded a significantly greater number of days of administration of morphine sulfate in the surgical NEC group compared with medical NEC group. These results are consistent with research by Hällström et al. ${ }^{21}$ who also found a significant correlation between the length of administering morphine sulfate with the development of NEC. Our research has identified the link between the length of administration of morphine sulfate and the severity of NEC, through the observed fact that the preterm infants with the most severe stage of NEC (surgical NEC) received morphine sulfate longer compared with premature infants in the medical NEC group. Since the current available literature contains no studies that have thoroughly analyzed the effect of duration of receiving morphine sulfate to develop of NEC and its seriousness, we were not able to compare our results with the results of other studies. Validation of this observation in larger populations is warranted.

It is well known that some preterm infants with advanced NEC or sepsis have evidence of circulatory failure and microvascular dysfunction, often presenting with low blood pressure and high cardiac output with the need for using inotropes to maintain cardiorespiratory function. ${ }^{22-24}$ Although inotropes (dopamine or dobutamine) widely used in circulatory failure secondary to sepsis or NEC, combined with adequate ventilatory support, there is still disagreement about their influence on intestinal perfusion or their relationship with NEC. Hentschel et al. ${ }^{25}$ found that the administration of inotropes had no negative influence on superior mesenteric artery blood flow during continuous infusion, but other data in the literature are limited and mostly derived from animal rather than human studies. However, there is evidence that the circulatory physiology in the preterm infants is distinct from that seen at any other time with the reported cases that some premature infants may have decreased cardiac adrenergic receptors and innervation, meaning that these infants may have an impaired response to inotropic intervention. ${ }^{26}$ The results of our study showed a statistically significant association between administration of inotropes in subjects with NEC compared to the control group. These results can be observed in the context of pathophysiological events and endpoints in advanced cases of NEC in preterm infants.

All of the above findings must be observed through the prism of prematurity with the mutual synergy of various immature organic systems. Preterm infants born at less than 28 to 30 weeks of gestation have very fragile lung tissue with a great tendency to lung injury and the consequent tendency to trap air, collapse, or fill with mucus and other fluids, which further compromise lung growth and development. ${ }^{27}$ This condition known as bronchopulmonary dysplasia (BPD) has a significant influence on the increased frequency of use of mechanical ventilation and administration of morphine sulfate in premature infants with NEC. Preterm infants with BPD also have nutritional and fluid problems because of fluid sensitivity and increased metabolic needs and show vulnerability to infections, especially respiratory infections. ${ }^{28}$ Therefore, it is justified that these clinical variables should be considered in the same context.

This study has some limitations, such as the inherent errors and bias of retrospective 
nature of the study, relatively small patient population and restriction to a single pediatric surgical center in a relatively small geographic area in Bosnia and Herzegovina as well as inability to calculate population-based rates.

Even with the above-mentioned limitations, we can conclude that knowledge of the risk factors associated with the development of NEC can contribute to more efficient prevention of this devastating acquired disease. Recognizing the potential impact of common treatment given to premature infants in the NICU's and modifications of its application, including selective use of opioids based on clinical judgment and evaluation of pain indicators as well as replacement of intubation and mechanical ventilation with non-invasive positive-pressure ventilation techniques, especially continuous positive airway pressure, can reduce the complications associated with long-term invasive ventilation and longer administration of morphine sulfate.

\section{REFERENCES}

1. Hunter CJ, Upperman JS, Ford HR, Camerini V. Understanding the susceptibility of the premature infant to necrotizing enterocolitis (NEC). Pediatr Res 2008; 63: 117-123.

2. Zvizdic Z, Heljic S, Popovic N, Alajbegovic-Halimic J, Milisic E, Jonuzi A. Contributing factors for development of necrotizing enterocolitis in preterm infants in the Neonatal Intensive Care Unit. Mater Sociomed 2016; 28: 53-56.

3. Stewart CJ, Marrs ECL, Magorrian S, et al. The preterm gut microbiota: changes associated with necrotizing enterocolitis and infection. Acta Paediatr 2012; 101: 1121-1127.

4. Neu J, Walker WA. Necrotizing enterocolitis. N Engl J Med 2011; 364: 255-264.

5. Aranda JV, Carlo W, Hummel P, Thomas R, Lehr VT, Anand KJ. Analgesia and sedation during mechanical ventilation in neonates. Clin Ther 2005; 27: 877-899.

6. Anand KJ. Clinical importance of pain and stress in preterm neonates. Biol Neonate 1998; 73: 1-9.

7. Bellù R, de Waal K, Zanini R. Opioids for neonates receiving mechanical ventilation: a systematic review and meta-analysis. Arch Dis Child Fetal Neonatal Ed 2010; 95: F241-F251.

8. Playfor S, Jenkins I, Boyles C, et al; United Kingdom Paediatric Intensive Care Society Sedation; Analgesia and Neuromuscular Blockade Working Group. Consensus guidelines on sedation and analgesia in critically ill children. Intensive Care Med 2006; 32: $1125-1136$.
9. Menon G, Anand KJS, McIntosh N. Practical approach to analgesia and sedation in the neonatal intensive care unit. Semin Perinatol 1998; 22: 417-424.

10. Soliman HM, Melot C, Vincent JL. Sedative and analgesic practice in the intensive care unit: the results of a European survey. Br J Anaesth 2001; 87: 186-192.

11. Anand KJ, Barton BA, McIntosh N, et al. Analgesia and sedation in preterm neonates who require ventilatory support: results from the NOPAIN trial. Neonatal Outcome and Prolonged Analgesia in Neonates. Arch Pediatr Adolesc Med 1999; 153: 331-338.

12. Bell MJ, Ternberg JL, Feigin RD, et al. Neonatal necrotizing enterocolitis. Therapeutic decisions based upon clinical staging. Ann Surg 1978; 187: 1-7.

13. Walsh MC, Kliegman RM. Necrotizing enterocolitis: Treatment based on staging criteria. Pediatr Clin North Am 1986; 33: 179-201.

14. Dolgin SE, Shlasko E, Levitt MA, et al. Alterations in respiratory status: Early signs of severe necrotizing enterocolitis. J Pediatr Surg 1998; 33: 856-858.

15. Ostermann ME, Keenan SP, Seiferling RA, Sibbald WJ. Sedation in the intensive care unit: a systematic review. JAMA 2000; 283: 1451-1459.

16. Anand KJ, Hall RW. Pharmacological therapy for analgesia and sedation in the newborns. Arch Dis Child Fetal Neonatal Ed 2006; 91: F448-F453.

17. Simons SH, van Dijk M, van Lingen RA, et al. Routine morphine infusion in preterm newborns who received ventilatory support: a randomized controlled trial. JAMA 2003; 290: 2419-2427.

18. Saarenmaa E, Neuvonen PJ, Rosenberg P, Fellman V. Morphine clearance and effects in newborn infants in relation to gestational age. Clin Pharmacol Ther 2000; 68: 160-166.

19. Cignacco E, Hamers JP, van Lingen RA, et al. Pain relief in ventilated preterms during endotracheal suctioning: a randomized controlled trial. Swiss Med Wkly 2008; 138: 635-645.

20. Hällström M, Koivisto AM, Janas M, Tammela O. Frequency of and risk factors for necrotizing enterocolitis in infants born before 33 weeks of gestation. Acta Paediatr 2003; 92: 111-113.

21. de Waal K, Evans N. Hemodynamics in preterm infants with late-onset sepsis. J Pediatr 2010; 156: 918-922.e1.

22. Seri I. Circulatory support of the sick preterm infant. Semin Neonatol 2001; 6: 85-95.

23. Cox DJ, Groves AM. Inotropes in preterm infants-evidence for and against. 2012; 101 (Suppl 464): 1723.

24. Hentschel R, Hensel D, Brune T, Rabe H, Jorch G. Impact on blood pressure and intestinal perfusion of dobutamine or dopamine in hypotensive preterm infants. Biol Neonate 1995; 68: 318-324. 
25. Kluckow M. Low systemic blood flow and pathophysiology of the preterm transitional circulation. Early Hum Dev 2005; 81: 429-437.

26. Smith VC, Zupancic JAF, McCormick MC, et al. Trends in severe bronchopulmonary dysplasia rates between 1994 and 2002. J Pediatr 2005; 146: 469473.
27. Vaucher YE. Bronchopulmonary dysplasia: An enduring challenge. Pediatr Rev 2002; 23: 349-358. 